\title{
Chronic flexor tenosynovitis: missed diagnosis for a decade
}

\author{
Linda Morris, Alan Stevens, Michael Doherty
}

Rheumatology Unit, City Hospital, Nottingham

L Morris

M Doherty

\section{Pathology}

Department, Queen's Medical Centre, Nottingham A Stevens

Correspondence to: Professor M Doherty Professor M Doherty, Rheumatology Unit, City NG5 1PB United Kingdom.

Accepted for publication 21 March 1996

\section{Case report}

A 21 year old white medical student asked for informal advice while she was attached to the rheumatology module. She gave a 10 year history of chronic pain and stiffness affecting the index finger of her left hand, with swelling of the proximal volar aspect of the finger. Over the last year she had noticed increasingly frequent triggering and difficulty with precision movements of the left hand. She was otherwise fit and well.

Examination revealed proximal flexor tenosynovial swelling of the index finger with fine crepitus on movement. No obvious tendon nodule was palpable. Nothing else was apparent on locomotor or general examination.

Her only relevant past history was a hand injury at 10 years of age. She had been swimming in Hong Kong when she fell onto a sea urchin with her outstretched left hand. Her parents removed all visible spines but within 24 hours she had an acutely painful swollen left hand. She was taken to casualty and given antibiotics for presumed infection. The swelling quickly resolved and by two weeks her hand was normal again. It was eight months later that she noted insidious onset of pain, stiffness, and swelling related to her left index finger. She was referred to a rheumatologist and subsequently an orthopaedic surgeon. Blood tests (full blood count, erythrocyte sedimentation rate, biochemistry) and a hand $x$ ray were normal at age 11. She was given a diagnosis of idiopathic (presumed traumatic) tenosynovitis. She had no triggering at that time and conservative management was advised. She had obtained no other specialist opinion until her current student attachment.

A radiograph of the left hand at age 21 was entirely normal, with no soft tissue swelling, calcification, periostitis, or pressure erosion. In view of her history she was referred to a hand surgeon who undertook tenosynovectomy of the flexor digitorum profundus tendon. At operation the tendon sheath appeared thickened with an irregular, roughened synovial lining. Within the tendon sheath was a minute calcareous fragment. Histology revealed inflamed and thickened synovium with a chronic inflammatory cell infiltrate and hyperplasia of the synovial lining cells (fig 1). A central core consisted of focally calcified mate- rial surrounded by giant cells (fig 2). Culture was negative at six weeks.

There were no complications following surgery and two years later the patient is asymptomatic with normal hand function.

\section{Discussion}

The principal differential diagnosis of this student's chronic monotenosynovitis lay between penetrating foreign body injury ${ }^{1}$ and pigmented villonodular tenosynovitis (PVNS). The history of preceding sea urchin spine injury clearly offered a cause of the former diagnosis, though plant thorn tenosynovitis following a forgotten penetrating injury ${ }^{2}$ remained a possibility. Chronic infection (for example, atypical mycobacteria) was less likely, given the 10 year history and normal

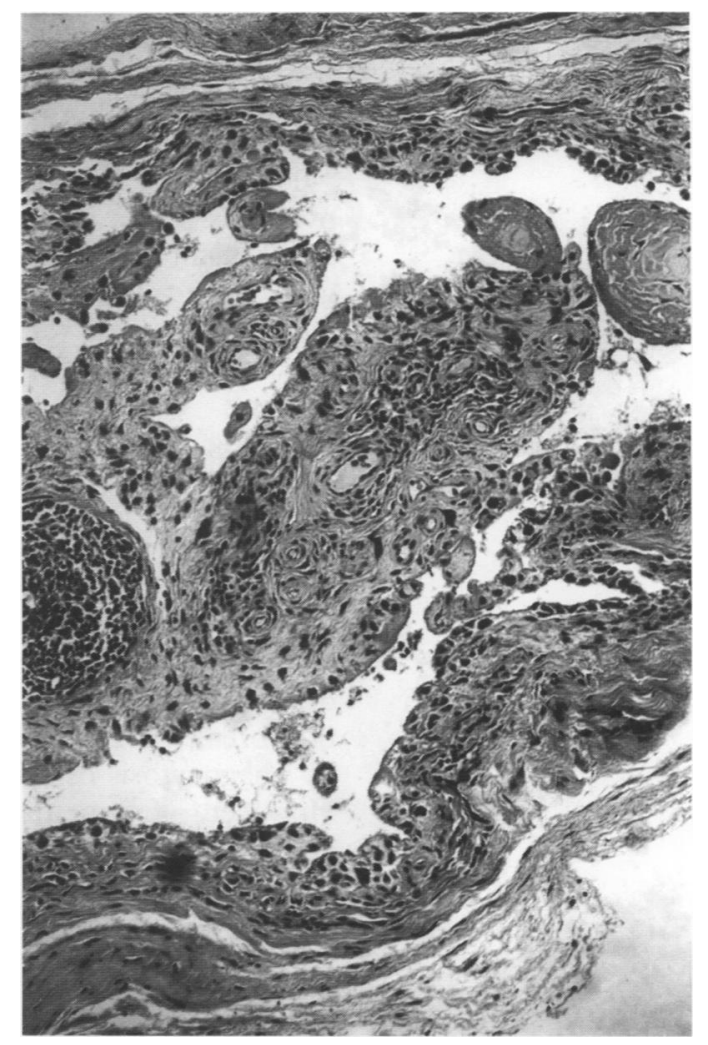

Figure 1 Photomicrograph of thickened synovium of flexor tendon sheath showing focal lymphocytic infiltrate, fibrovascular thickening of synovial support tissue, hyperplasia of synovial epithelial cells, and occasional spherical masses of old fibrin. 


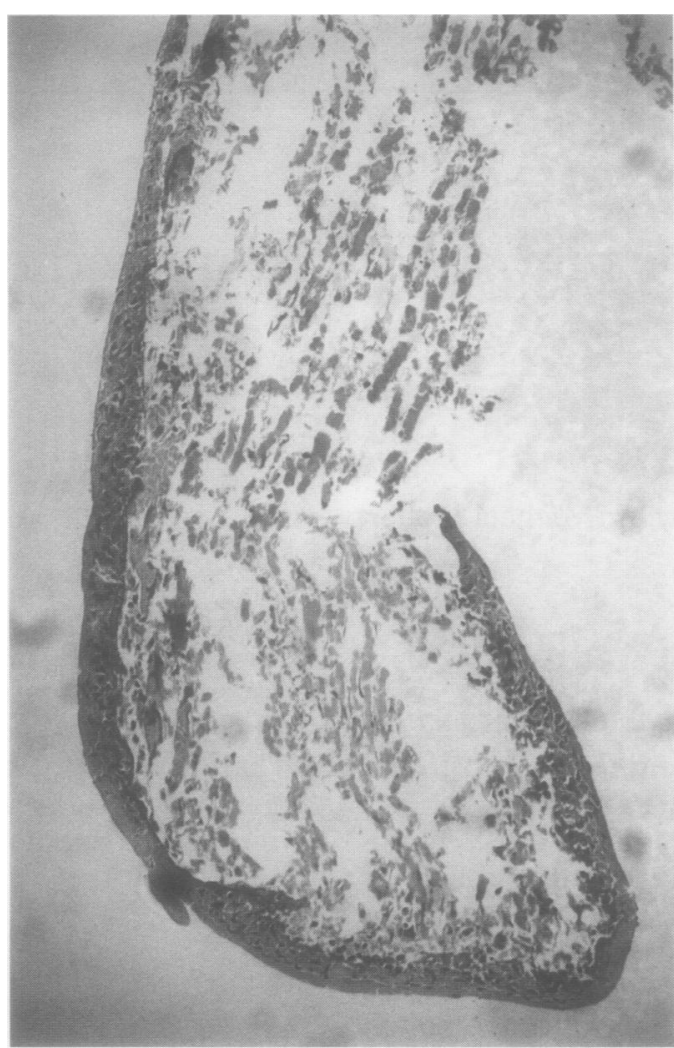

Figure 2 High power photomicrograph of the longitudinal section through the decalcified foreign body showing remnants of its central crystalline structure and its outer coating of old fibrin containing mixed chronic inflammatory cells.

radiograph. She was undertaking no unusual repetitive activity to cause chronic traumatic tenosynovitis, and had no overlying callus or skin change. Imaging and biopsy were the key investigations; her symptoms and disability warranted total excision of abnormal tenosynovium. Histology was non-specific but consistent with urchin spine synovitis. ${ }^{13}$

Penetrating foreign body injury is often overlooked as a cause of monosynovitis. ${ }^{1}$ The usual result of sea urchin spine injury is negligible, with transient but often intense stinging pain. ${ }^{34}$ However, variable local and systemic reactions are described ${ }^{3-8}$ including acute self limiting synovitis or tenosynovitis with overlying erythema. ${ }^{15}$ As with plant thorn synovitis $^{12}$ such florid inflammation may be misdiagnosed as infection and treated as such; spontaneous resolution within several days of antibiotic treatment reinforces this misdiagnosis. Sepsis, however, appears a rare complication $^{35-8}$ which may prove resistant to oral antibiotics. ${ }^{8}$ Small portions of the spine that break off in soft tissues may be absorbed by phagocytosis or migrate and be expelled. ${ }^{34}$ However, fragments that are deep or embedded in firm tissue such as tendon or capsule may remain to stimulate a chronic granulomatous inflammation, with recurrence of symptoms several months or even years after the initial injury. ${ }^{13-5}$ This may be considered a new clinical problem if the initial incident is forgotten or its relevance overlooked, as in our medical student. Chronic sequelae that may result include synovitis, ${ }^{347}$ tenosynovitis, ${ }^{23}$ granulomata, ${ }^{56}$ and peripheral nerve entrap- ment. ${ }^{13}$ If a chronic granulomatous reaction develops it is likely to persist until surgically removed. ${ }^{134}$

Embedded sea urchin spines may be visible on radiographs, though small fragments are usually not seen. ${ }^{3}$ Even with established synovitis, bony change and cartilage loss are usually absent. ${ }^{135}$ The hand is a common site of involvement. The purple sea urchin Strongylocentrotus purpuratus is a common culprit, occurring in high density along the west coast of North America. The mechanism of sea urchin spine inflammation is uncertain. The spine is composed of a central calcium carbonate (calcite) rod encased by epithelium. Calcite has a regular repeating crystalline structure (fig 3) and, like the semi-crystalloid blackthorn and date palm tips, may act as inflammatory extrinsic crystals or particles. ${ }^{19}$ Alternatively the protein epithelium may possess phlogistic potential. $^{5}$

In Europe plant thorns, especially blackthorn and rose, are the more usual extrinsic agents to cause acute and chronic synovitis. ${ }^{2}$ Nevertheless, with increased foreign travel
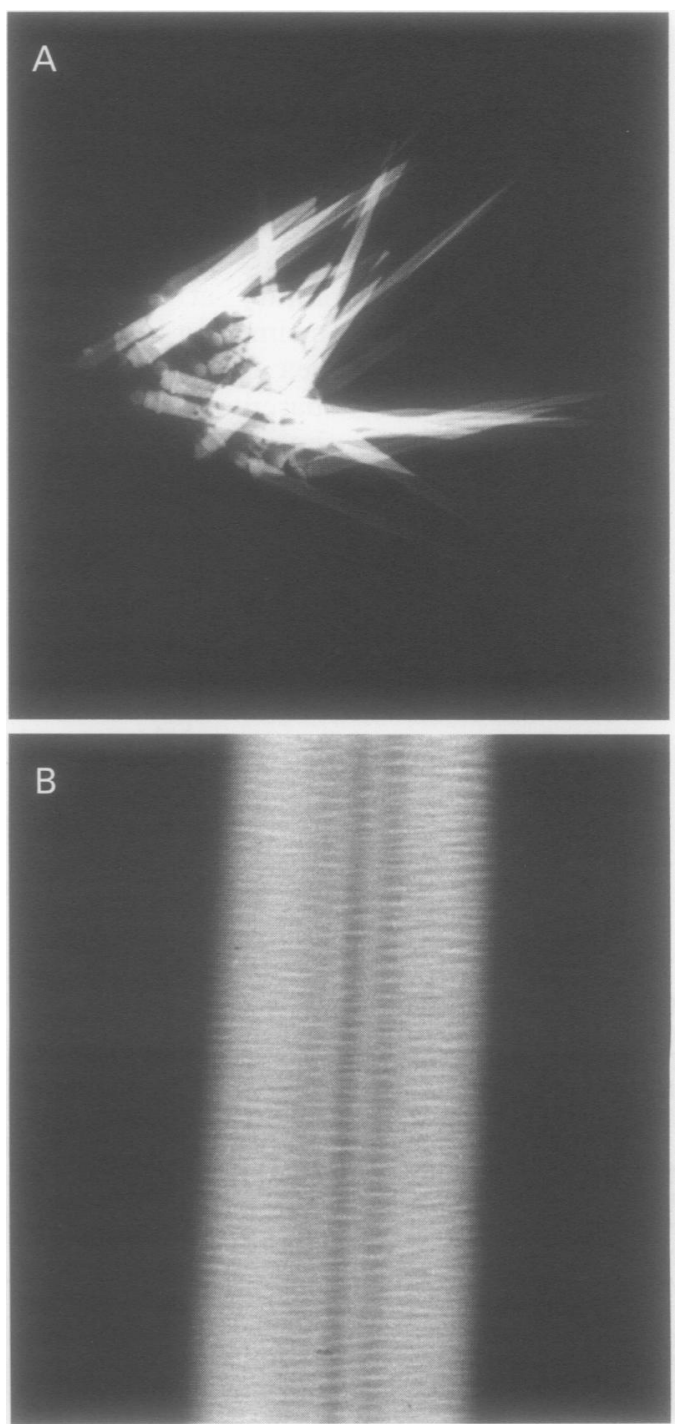

Figure 3 (A) Radiograph of sea urchin spines (Strongylocentrotus purpuratus). The highly magnified view $(B)$ shows the ordered arrangement of the crystalline calcite component. 
more exotic injury from sea urchin spines, date palm fronds, and cactus ${ }^{15}$ may need to be considered.

\section{The lesson}

- The acute inflammation of penetrating spine or thorn injury is often self-limiting and misdiagnosed as sepsis.

- The history of prior injury may be forgotten or overlooked at the time of presentation with chronic synovitis

- Investigations are usually unhelpful.

- Surgical removal is necessary for diagnosis and is curative.

Since the Editor is one of the coauthors, Dr Janet McDonagh served as Acting Editor to arrange the impartial review of this paper.
1 Reginato AJ, Ferreiro JL O' Connor CR, Barbasan C, Arasa $\mathrm{J}$, Bednar J, et al. Clinical and pathological studies of twenty-six patients with penetrating foreign body injury to the joints, bursae and tendon sheaths. Arthritis Rheum 1990;33:1753-62.

2 Kelly JJ. Blackthorn inflammation. F Bone foint Surg 1966;3:474-7.

3 O'Neal RL, Halstead BW, Howard LD. Injury to human tissues from sea urchin spines. Calif Med 1964;101:199 202.

4 Strauss MB, MacDonald RI. Hand injuries from sea urchin spines. Clin Orthop Rel Res 1976;114:216-8.

5 Cracchiolo AC, Goldberg L. Reaction to puncture injuries by the sea urchin spine and date palm thorn. Arthritis Rheum 1977;20:1206-12.

6 Kinmont PDC. Sea urchin sarcoidal granuloma. $\mathrm{Br}$ Dermatol 1965;77:335-42.

7 Moynahan EJ, Montgomery PR. Echinoderm Granuloma. Br ₹ Clin Prac 1968;22:265-9.

8 Daupleix D, Dreyfus J, Amouroux J. Sea-urchin spine synovitis. Rev Rheum 1982;49:219-20.

9 Doherty M, Whicher JT, Dieppe PA. Activation of the alternative pathway of complement by monosodium urate monohydrate crystals and other inflammatory particles. Ann Rheum Dis 1983;42:285-91. 